\title{
The genetics of conidiophore pigmentation in Aspergillus nidulans
}

\author{
A. John Clutterbuck \\ Department of Genetics, Glasgow University, Glasgow G11 5JS, UK
}

(Received 19 February 1990; revised 24 April 1990; accepted 11 June 1990)

\begin{abstract}
The grey-brown pigmentation of Aspergillus nidulans conidiophores depends on the functions of two 'ivory' loci. ivo $B$ codes for a developmental specific phenol oxidase, and mutants accumulate its substrate $N$-acetyl-6hydroxytryptophan. ivo $A$ mutants are unable to make this substrate. yg $A$ mutants are also poorly pigmented, and extracts require copper salts to activate both the phenol oxidase and conidial laccase. $i v o A$ and ivo $B$ mutants partially suppress the spore colour phenotype of $y g A$ mutants. Comparisons of morphology, phenol oxidase and substrate accumulation in morphological mutants at the $b r l A$ locus suggest that the $b r l A$ protein regulates ivoA, $i v O B$ and morphogenetic loci independently. The med $A$ locus, which also affects morphology and pigmentation, may code for a modifier of $b r l A$ function. $a b a A$ mutants which are blocked at a later stage of development than $b r l A$ or med $A$ mutants have low phenol oxidase levels, implying that by this stage of development the activity of the $i v o B$ locus is declining.
\end{abstract}

\section{Introduction}

The conidiophores of the filamentous ascomycete Aspergillus nidulans are pigmented grey-brown. This is not conspicuous in wild-type strains, but is visible in aconidial mutants (Clutterbuck, 1969) such as 'abacus' $(a b a A)$ or 'bristle' $(b r l A)$, in which mutant derivatives of the conidiophores, metulae and phialides are exposed. 'Ivory' (ivo) mutants, which have lost this pigment, have been isolated in $a b a A$ or $b r l A$ mutant backgrounds (Clutterbuck, 1969) and details of their properties and interactions with other developmental mutants are presented here. A specific phenol oxidase (AHTase), lacking in ivoB mutants, is described in the accompanying paper (Birse \& Clutterbuck, 1990a) and its substrate has been identified as $N$-acetyl-6-hydroxytryptophan (AHT; McCorkindale et al., 1983).

Summaries of this work have previously been included in reviews of $A$. nidulans conidiation (Clutterbuck, 1977, 1978, 1990a).

\section{Methods}

Media and general methods for Aspergillus genetics are described by Pontecorvo et al., (1953) and Clutterbuck (1974). All strains were

Abbreviations: AHT, $\mathrm{N}$-acetyl-6-hydroxytryptophan; AHTase, $\mathrm{N}$ acetyl-6-hydroxytryptophan oxidase. derivatives of those in the Glasgow collection (Clutterbuck, 1986), and all carried the veA1 mutation. ivo mutants were induced in biAl abaA6, biAl abaA20 or biAl brlA42 strains, using ultraviolet light or $N$-methyl$N^{\prime}$-nitro- $N$-nitrosoguanidine as described by Clutterbuck (1969). Other genetic markers are listed in Clutterbuck $(1990 \mathrm{~b})$. Top layer cultures on complete medium agar (Clutterbuck, 1972) were used for the growth of mycelium for extraction of AHT and AHTase: $9 \mathrm{~cm}$ plates containing $20 \mathrm{ml}$ of agar medium were overlaid with $4 \mathrm{ml}$ of liquid complete medium containing an inoculum of $10^{4}$ conidia or ascospores per dish. Plates were incubated on a level incubator shelf at $37^{\circ} \mathrm{C}$.

AHT was extracted as described by McCorkindale et al. (1983), and assayed using the acid-diazotized sulphanilic acid test for 6-hydroxyindoles (Jepson, 1960; Jepson et al., 1962). A $0.1 \mathrm{ml}$ volume of a $5 \%$ (w/v) aqueous solution of $\mathrm{NaNO}_{2}$ was added to $0.1 \mathrm{~g}$ sulphanilic acid dissolved in $1 \mathrm{ml}$ concentrated $\mathrm{HCl}$, and left for $5 \mathrm{~min}$ on ice. Excess nitrous acid was destroyed by adding a crystal of ammonium sulphamate. Then $0.1 \mathrm{ml}$ of the reagent was added to $2.8 \mathrm{ml} 1 \mathrm{M}-\mathrm{HCl}$ and $0.1 \mathrm{ml}$ of the aqueous extract to be assayed and left for $20 \mathrm{~min}$ in the dark. $A_{530}$ was read in a Pye-Unicam spectrophotometer, subtracting $\left(0.55 \times A_{620}+0.45 \times A_{420}\right)$ background. Results are expressed as total absorbance per dish. Colonies on plates were tested for 6hydroxyindoles by overlaying with filter paper soaked in the reagent.

AHT and other indoles were detected by paper chromatography of methanolic extracts of dried mycelium (Jepson, 1960), using butanol/ acetic acid/water $(60: 15: 25$ by vol.) solvent and stained with Ehrlich's reagent or diazotized sulphanilic acid.

AHTase is an extracellular enzyme, extractable by shaking mycelium with buffer. This procedure, and the colorimetric assay using hydroquinone monomethyl ether as substrate, are as described by Birse $\&$ Clutterbuck $(1990 a)$. In the medA/medA $A^{+}$comparison $10 \mathrm{mM}^{-}$ phosphate buffer (pH 7.0) was substituted for ammonium tartrate. Tests of AHTase thermolability and electrophoretic mobility are also described by Birse and Clutterbuck (1990a). 


\section{Results}

ivo mutants

A total of 200 ivo ('ivory' - unpigmented conidiophore) mutants have been isolated in $A$. nidulans as described in Clutterbuck (1969), starting with an $a b a A$ or brlA42 aconidial background in which the conidiophore is directly visible. The ivo mutants had no evident effect on either growth or morphology.

Complementation tests performed by stabbing strains close together on complete medium divided the mutants into two main classes: ivo $A$ and ivoB. ivo $A$ mutants were approximately seven times commoner than $i v o B$. A third class of partially defective mutants failed to complement in diploids with $y g A 6$. This mutant is characterized by yellow-green spore pigment, and is believed to be deficient in copper distribution (Clutterbuck, 1972).

The pattern of pigmentation at the junctions of complementing colonies suggested that a diffusible material accumulated in $i v o B$ mutants would cross-feed, and produce pigmentation in ivo $A$ strains. We have purified this material and identified it as $\mathrm{N}$-acetyl-6hydroxytryptophan (AHT; McCorkindale et al. 1983). Accumulation of this compound in $i v o B$ and $y g A$ mutants is associated with a deficiency of a specific phenol oxidase ( $\mathrm{N}$-acetyl-6-hydroxytryptophan oxygenase AHTase) which converts the substrate to a melanin (Birse \& Clutterbuck, 1990a). These results are summarized in Table 1.

Genetics of ivo $A$, ivo $B$ and $y g A$ mutants. Fig. 1 represents regions of the $A$. nidulans linkage map to which these three mutations have been located. These data have been summarized by Clutterbuck (1990 b).

The $a d D$ to $y g A$ linkage was determined using two new $y g A$ alleles ( $y g A 7$ and $y g A 8)$ induced in an $a d D 3$ strain. The resulting strains were crossed with a $\operatorname{trp} A$ strain, selecting for $\mathrm{ad}^{+}$(Clutterbuck, 1981). Five out of more than 5000 colonies were yellow-green, and four of these were $\operatorname{trp} A$, implying that $\operatorname{trp} A$ and $y g A$ are on opposite sides of $a d D$.

The $i v o B$ to $u r e D$ linkage was obtained from a cross between the two single mutants in which no recombinants were obtained among 400 fully analysed colonies plus more than 1000 colonies plated selectively for $u r e D^{+}$.

The ivoB locus. These mutants lack AHTase and accumulate its substrate, AHT (Table 1). Evidence that $i v o B$ is the structural gene for AHTase was provided by a temperature-sensitive mutant ivoB192 which had an 'ivory' phenotype at $37^{\circ} \mathrm{C}$, but was more or less wild-type at $25^{\circ} \mathrm{C}$. This mutant was shown to be allelic to other ivo $B$ mutants by complementation and recombination tests, and enzyme extracts from the mutant were more thermolabile than wild-type enzyme (Birse \& Clutterbuck, $1990 a$ ).

ivo $B$ mutants accumulate AHT (see Fig. 4), and to a lesser extent small quantities of a compound tentatively identified as 6-hydroxytryptophan (McCorkindale $e t$ al. 1983). Attempts were made to alter AHT accumulation by $i v o B$ strains by supplementing the medium: acetate, leucine, anthranilic acid, indole, mevalonolactone, phenylalanine and tyrosine all failed to increase AHT accumulation, and L-tryptophan and DL- $N$-acetyltryptophan significantly decreased AHT yield.

The ivoA locus. Mutants at this locus possess AHTase, but are assumed to be defective in biosynthesis of AHT, since they fail to accumulate it when in combination with an $i v o B$ mutation. Chromatograms of extracts of ivo $A$ ivo $B$ double mutants revealed no 6-hydroxyindoles identifiable with diazotized sulphanilic acid, nor did they show any conspicuous indole staining with Ehrlich's reagent. A spot corresponding to $N$-acetyltryptophan was sometimes seen, but was not conspicuous. A tryptophan spot was detectable in all extracts and sometimes appeared more intense in those from ivo $A$ strains.

ivo $A$ mutants have approximately $60 \%$ more AHTase activity than $i v o A^{+}$strains (compare $b r l A 7$ and $b r l A 7$

Table 1. Phenotypes of mutants affecting Aspergillus conidiophore pigmentation

+ , Presence; $(+)$, presence at reduced level; - , absence.

\begin{tabular}{lcccc}
\hline \hline Mutant & Pigment & AHTase & $\begin{array}{c}\text { AHT } \\
\text { accumulation }\end{array}$ & $\begin{array}{c}\text { AHT accumulation } \\
\text { in ivo } \\
\text { background }\end{array}$ \\
\hline Wild-type & + & + & - & + \\
ivo $A$ & - & + & - & - \\
ivo $B$ & $(+)$ & $(+)$ & + & + \\
$y g A$ & $(+)$ & & + \\
\hline \hline
\end{tabular}


Linkage group III

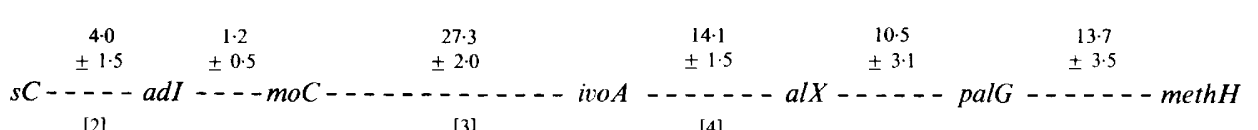

[2]

[3]

[4]

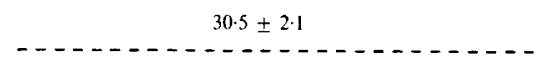

[5]

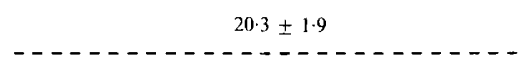

[4]

Linkage group II

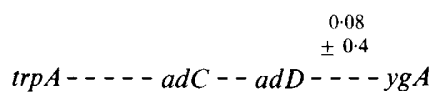

Linkage group VIII

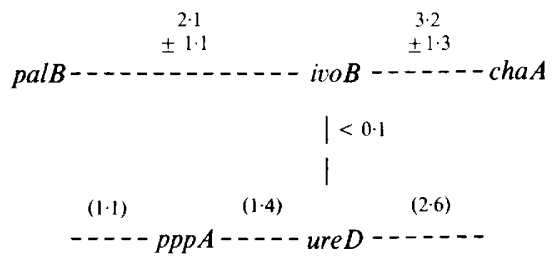

Fig. 1. Linkage map showing positions of $i v o A, i v o B$ and $y g A$ loci. The data are derived from single crosses except as indicated by numbers in square brackets. Linkage distances are percentages of recombinants \pm standard error. Figures in parentheses are linkage estimates from Hankinson (1974) (in which ureD $=u Z$ ).

Table 2. AHTase activities of $y g A$ strains assayed in the presence of copper salts

Two other ivoB mutants gave similar results to those of the brlA42; ivoB63 strain. Results are expressed as $100 \times \Delta A_{470} \mathrm{~min}^{-1}$ per culture dish. Figures are the means of duplicate assays.

\begin{tabular}{|c|c|c|c|c|c|c|c|}
\hline \multirow[b]{2}{*}{ Strain } & \multirow{2}{*}{$\begin{array}{l}\text { Copper } \\
\text { salt }\end{array}$} & \multicolumn{6}{|c|}{ Copper concn (mM) } \\
\hline & & 0 & $0 \cdot 1$ & $0 \cdot 3$ & $1 \cdot 0$ & $3 \cdot 0$ & $10 \cdot 0$ \\
\hline brlA42 & $\mathrm{CuCl}$ & $51 \cdot 2$ & $53 \cdot 6$ & $46 \cdot 8$ & $54 \cdot 8$ & $51 \cdot 6$ & 0.4 \\
\hline brlA42; ivoB63 & $\mathrm{CuCl}$ & $3 \cdot 2$ & $2 \cdot 0$ & $4 \cdot 0$ & 4.4 & $4 \cdot 8$ & 0.0 \\
\hline brlA42;ygA6 & $\mathrm{CuCl}$ & $3 \cdot 2$ & 7.6 & $15 \cdot 2$ & $21 \cdot 2$ & $27 \cdot 6$ & 0.0 \\
\hline brlA42; ygA6 & $\mathrm{CuSO}_{4}$ & $3 \cdot 2$ & - & $12 \cdot 4$ & $16 \cdot 0$ & $19 \cdot 6$ & $17 \cdot 6$ \\
\hline
\end{tabular}

ivo $A 1$ in Table 3). It seems probable that in ivo $A^{+}$strains tanning destroys some of the AHTase enzyme by incorporating it into a melanin complex, but that this does not occur in the absence of substrate. A similar phenomenon has been seen with the spore pigment enzyme laccase I (Clutterbuck, 1972; Kurtz \& Champe, 1982). The $K_{\mathrm{m}}$ and electrophoretic properties of the enzyme were unaffected by ivo $A$ (data not shown).

The ygA locus. AHTase, like other phenol oxidases (Malström \& Rydén, 1968), contains copper and, less typically, zinc (Birse \& Clutterbuck, 1990a). Mutants at the $y g A$ locus are partially deficient in both conidial laccase (Clutterbuck, 1972) and AHTase. It is possible to restore laccase activity to extracts of a $y g A$ mutant (Clutterbuck, 1972), or a $y B$ mutant (Kurtz \& Champe, 1981 ) by addition of copper salts. Table 2 similarly demonstrates the restoration of AHTase activity to extracts of a $y g A$ mutant by incorporation of copper salts in the assay mixture. Both cuprous chloride and cupric sulphate significantly activated the $y g A$ extract, but not extracts of wild-type or $i v o B$ controls. The cuprous salt, however, was inhibitory at high concentrations. The restored AHTase activity was maintained after dialysis against buffer (data not shown).

Analysis of the progeny of appropriate crosses demonstrates that ivo mutants (both $A$ and $B$ ) partially suppressed the yellow-green conidial phenotype of $y g A$. 
Table 3. AHTase and AHT accumulation in morphological mutants

Colonies were grown for $3 \mathrm{~d}$ at $37^{\circ} \mathrm{C}$ from point inocula on $\mathrm{CM}$ agar. All assays are the means of two or more replicate colonies. AHTase and AHT levels are expressed as percentages relative to brlA42. The AHTase level in the brlA42 ivoB63 strain, and in brlA $1-3$, is taken as background activity from other phenol oxidases and spontaneous oxidation. The differences between brlA42 medA15 and brlA42 were assessed on two independent recombinants of each genotype: the differences in both AHTase activity and AHT accumulation are significant at the 0.05 probability level. Similarly, the brlA7/brlA7 ivoAl difference in AHTase was tested on 14 recombinants of each genotype, and was significant at the 0.001 probability level. Missing entries (-) were untested. Morphologies are coded according to the lettering of Fig. 2.

\begin{tabular}{|c|c|c|c|c|}
\hline $\begin{array}{l}\text { Relevant } \\
\text { genotype }\end{array}$ & Morphology & $\begin{array}{l}\text { Conidiophore } \\
\text { pigment }\end{array}$ & AHTase & $\begin{array}{c}\text { AHT in ivoB63 } \\
\text { derivative }\end{array}$ \\
\hline Wild-type & $e$ & + & $16 \cdot 5$ & $34 \cdot 3$ \\
\hline brlA1 & $a$ & - & $7 \cdot 2$ & - \\
\hline brla2 & $a$ & - & 7.7 & $0 \cdot 0$ \\
\hline brla3 & $a$ & - & $7 \cdot 0$ & - \\
\hline brla14 & $a$ & - & $56 \cdot 6$ & $2 \cdot 9$ \\
\hline brlA 19 & $a$ & - & 84.9 & 0.0 \\
\hline brlA6 & $a-b$ & + & 99.0 & $18 \cdot 3$ \\
\hline brla10 & $b$ & - & 71.9 & $2 \cdot 4$ \\
\hline brlA9 & $b$ & + & $134 \cdot 4$ & 43.4 \\
\hline brla 7 & $b-c$ & + & $192 \cdot 2$ & $22 \cdot 8$ \\
\hline brla35 & $b-c$ & + & 157.9 & $56 \cdot 1$ \\
\hline brlA42 & $c$ & + & 100 & 100 \\
\hline$a b a A l$ & $d$ & + & $25 \cdot 9$ & - \\
\hline$a b a A 6$ & $d-e$ & + & 17.5 & $58 \cdot 8$ \\
\hline$a b a A 20$ & $d$ & + & $21 \cdot 7$ & $81 \cdot 9$ \\
\hline brlA42 ivoB63 & $c$ & - & $9 \cdot 1$ & (100) \\
\hline brla42 medA15 & $c$ & $(+)$ & $192 \cdot 3$ & $57 \cdot 2$ \\
\hline brlA7 ivoAl & $b-c$ & - & $305 \cdot 6$ & 0.0 \\
\hline
\end{tabular}

On the other hand, mutants in $y A$, the structural gene for conidial laccase, did not affect the conidiophore pigmentation of $y g A$ mutants. It is possible that by abolishing conidiophore melanization, ivo mutants prevent the locking-up of copper resources by chelation. Tanning of the copper-containing AHT oxidase would also amount to a loss of copper, if this could otherwise have been recycled for use in conidial laccase. Since pigmentation of spores follows conidiophore pigmentation, it is to be expected that conidiophore pigment mutants would affect spore colour, but not vice versa.

The conidial colour of $y g A$ strains is dependent on the $\mathrm{pH}$ of the growth medium, being yellow (mutant) at high $\mathrm{pH}$ and green (wild-type) at low pH. The conidiophore pigmentation was less obviously responsive to $\mathrm{pH}$, but while the wild-type had approximately twice the AHT-

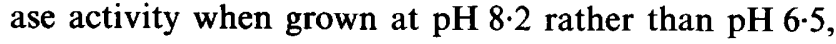
$y g A$ had similar activities on both media.

\section{Morphogenetic loci}

The brlA locus. Twenty-one null-phenotype mutants at the $b r l A$ locus develop undifferentiated bristles in place of conidiophores (Fig. 2a). These are unpigmented, and neither AHT nor AHTase is produced (see Table 3, brlA1-3 and Clutterbuck, 1969; Birse \& Clutterbuck, $1990 a$ ). In contrast, all brlA mutants (brlA6, 7, 9, 10, 12, $31,33,35,42)$ giving 'leaky' phenotypes, as judged by partial morphological progression, tested positively for AHTase (Table 3). These included two mutants (brlA 10see Table 3 ; and $b r l A 31$ - not tested quantitatively) whose conidiophores are not visibly pigmented. Furthermore, two mutants (brlA14 and brlA19) which show neither pigmentation nor morphological development were also AHTase positive.

AHTase extracted from leaky or temperature-sensitive brlA mutants (brlA9, 14, 35 and 42) was not distinguishable from the wild-type enzyme in thermolability or electrophoretic mobility.

Quantitative AHTase comparisons on 3-d-old colonies (Table 3) showed a range of activities, the highest coming from the brlA7, 9 and 35 mutants which have moderately leaky morphologies. Birse \& Clutterbuck $(1990 \mathrm{~b}$ ) found similar results for $26 \mathrm{~h}$ cultures assayed for both AHTase and ivoB mRNA.

Bristle mutants on their own accumulated only traces of AHT, if any, but the pigment precursor accumulated in ivoB63 recombinants at levels which correlated well with the degree of visible pigmentation. 


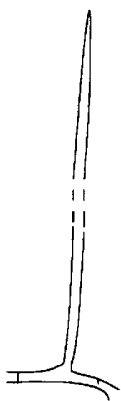

(a)

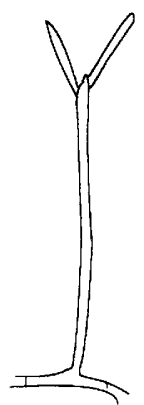

(b)

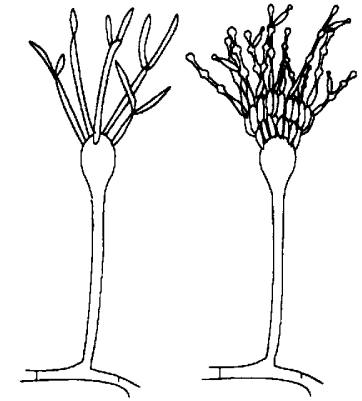

(c)

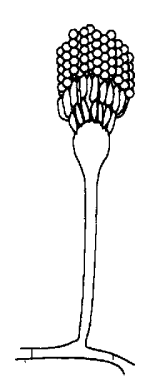

(e)
Fig. 2. Morphology of wild-type and $\mathrm{brlA}$ and $a b a A$ mutants (a) $\mathrm{brlA}$ null mutant ; $(b, c)$ increasingly leaky $b r l A$ mutants; $(d)$ aba $A$ mutant; $(e)$ wild-type.

The abaA locus. All abaA mutants contain AHTase, and none accumulate AHT. While AHTase levels were low in $a b a A$ mutants, approaching the level of the conidiating wild-type, substrate levels in the $i v o B$ derivatives were as high as for many leaky $b r l A$ mutants. The assays reported in Table 3 were made on whole colonies which would include conidiophores representing a spectrum of developmental stages. Low AHT or AHTase levels in the wild-type and $a b a A$ mutants therefore probably indicate down-regulation at the later stages of conidiation, whereas high levels in some $\operatorname{brlA}$ mutants indicate that these mutants are stuck at stages of maximum ivo $A$ and ivo $B$ activity.

The medA locus. Mutants at this locus have a partial 'bristle' phenotype in which a normal vesicle bears multiple layers of metulae surmounted by phialides and conidia (Clutterbuck, 1969). All mutants so far mapped to this locus exhibit only delayed conidiation. They also differ from $b r l A$ partial mutants in that the conidiophore pigmentation is reduced. In order to decide which component of the pigmentation system was deficient in medA mutants, a brlA42 medA15 strain was compared to a brlA42 control for AHTase levels, and a similar pair of strains, both carrying ivoB63, was used to assay AHT accumulation. Table 3 shows that medA strains had reduced AHT levels, and as in ivo $A$ mutants (see above), this was accompanied by increased AHTase.

\section{Developmental timing of AHT and AHTase production}

The production of AHTase by developing cultures has been shown to correspond closely to the time of formation of conidiophore vesicles (Clutterbuck, 1977), i.e. shortly before the conidiophore pigment becomes visible and about $4 \mathrm{~h}$ before the appearance of conidial laccase (Clutterbuck, 1972). Fig. 3 shows that accumu-

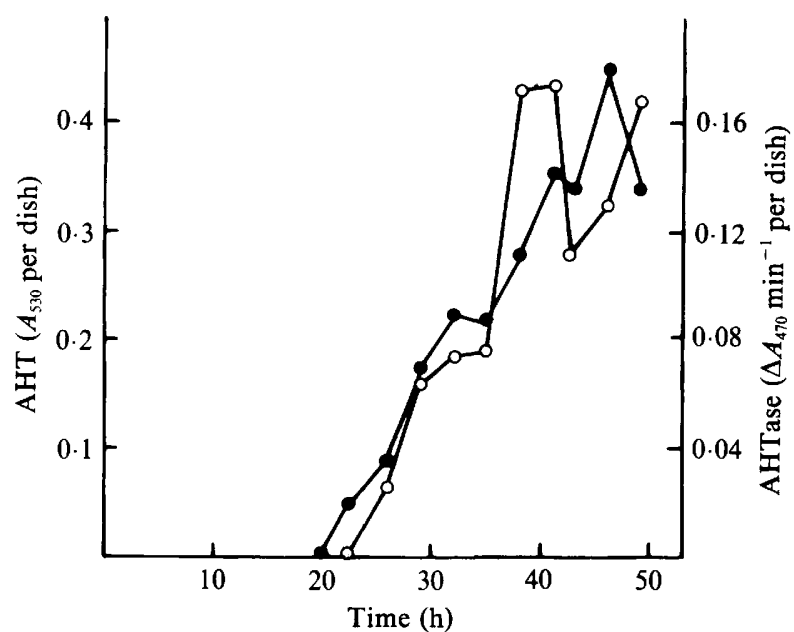

Fig. 3. Timing of AHT and AHTase formation by conidiating surface cultures of $A$. nidulans. Surface cultures were started from $10^{4}$ conidia per dish and incubated at $37^{\circ} \mathrm{C}$. Each point represents one dish. $\mathrm{O}$, AHT accumulated by a brlA42 ivoB63 strain;, AHTase extracted from a $\operatorname{brl} A 42$ strain.

lation of AHT in a brlA42 ivoB63 strain coincides with the rise in AHTase in a brlA42 $i v o B^{+}$strain.

\section{Discussion}

The conidiophore pigmentation system described here employs the fourth distinct phenol oxidase to be studied in $A$. nidulans. The other three, distinguished by substrate preference and developmental specificity, are hyphal tyrosinase (Bull \& Carter, 1973; Martinelli \& Bainbridge, 1974), conidial laccase I (Clutterbuck, 1972; Kurtz \& Champe, 1982) and cleistothecial laccase II (Hermann et al., 1983). Phenol oxidases in other fungi are similarly diverse, in both substrate utilization and function (Bell \& Wheeler, 1986).

In $A$. nidulans, conidial laccase I contributes to ultraviolet light resistance (Wright \& Pateman, 1970), and the other two systems are postulated to confer resistance to lysis (Kuo \& Alexander, 1967; Polacheck \& Rosenberger, 1977; Hermann et al., 1983). It is possible that conidiophore melanin might also provide some protection against ultraviolet light, but since the conidiophore is hardly pigmented before it becomes covered with conidia, this seems unlikely to be important.

The alternative role of melanins in consolidating polysaccharide walls against degradation, a function comparable to insect cuticle tanning (Andersen, 1985) or seed coat maturation (Marbach \& Mayer, 1978) seems more pertinent. While we have not demonstrated any structural deficiency in ivo mutants in morphologically normal conidiophores, pigmented $\operatorname{brl} A$ mutants have 


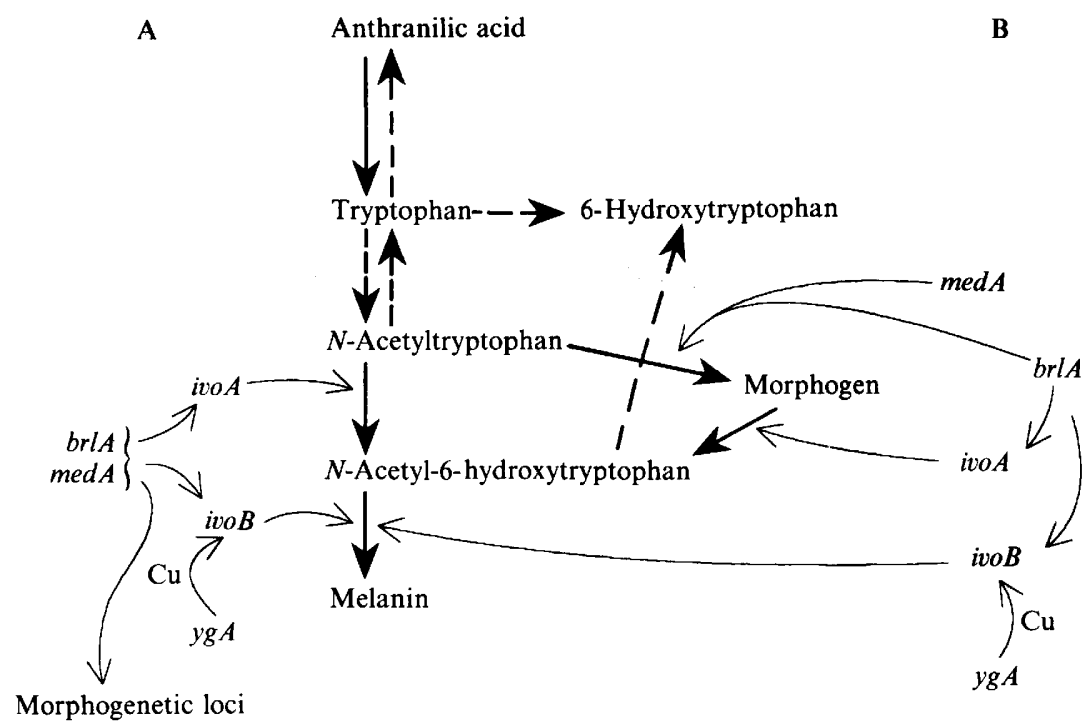

Fig. 4. Two possible schemes for the genetic control of conidiophore pigmentation and morphogenesis. Two alternative routes for the formation of the minor component 6-hydroxytryptophan are common to both schemes. Other features are discussed in the text. Degradation of tryptophan to anthranilic acid is shown by a broken arrow, signifying that it is postulated in scheme A to be induced by exogenous indoles. Interconversion of tryptophan and $\mathrm{N}$-acetyltryptophan are similarly assumed in scheme $\mathrm{A}$ to be conditional upon conidiation.

conspicuously more rigid bristles than unpigmented ones (see Fig. 5D and E in Birse \& Clutterbuck, 1990b).

Phenol oxidases are copper enzymes (Malström \& Rydén, 1968). The reactivation of AHTase activity in extracts of $y g A$ strains by the addition of copper is circumstantial evidence for the role of copper in this enzyme. However, AHTase is unusual among phenol oxidases in containing zinc as well as copper (Birse \& Clutterbuck, 1990 a), and although the necessity of zinc for AHTase activity remains to be tested, its presence may indicate an evolutionary origin distinct from other phenol oxidases.

To have a tryptophan derivative as principal substrate is unusual, although indoles are standard intermediates in the formation of melanin from tyrosine. We have suggested (Birse \& Clutterbuck, 1990a) that the final melanin incorporates other materials oxidized in secondary reactions with oxidized AHT, and we cannot exclude the possibility that AHTase also reacts with some of these directly.

The conidiophore pigmentation system is one aspect of Aspergillus development where we know something of the biochemistry behind the phenotype. We wish now to learn something about the developmental control of this process. It should first be noted that a simple induction pathway of AHTase by its substrate does not seem to operate, since ivo $A$ mutants, which lack AHT, nevertheless have high levels of AHTase.

A scheme integrating the morphogenetic and pigmen- tation pathways should explain the following observations.

1. Mutants at two loci, brlA and medA, have quantitatively correlated effects on pigmentation and morphogenesis.

2. ivo A mutants which are blocked in AHT synthesis do not accumulate the assumed precursor, $N$ acetyltryptophan.

3. Addition of tryptophan or $N$-acetyltryptophan to the medium has the effect of decreasing rather than increasing the level of AHT accumulated by an ivoB mutant.

4. Tryptophan auxotrophs grown on limiting tryptophan conidiate poorly and have relatively unpigmented conidiophores (Yelton et al., 1983).

5. We have found that a tryptophan auxotroph will not grow on $\mathrm{N}$-acetyltryptophan, but where tryptophan is limiting, addition of $N$-acetyltryptophan allows full conidiation.

Fig. 4 illustrates two possible schemes. Scheme A suggests that the product of medA modifies the action of $b r l A$, and thus plays a part in regulation of $i v o A, i v o B$ and unknown morphogenetic loci. The medA product might for instance be involved in post-translational modification of a $b r l A$-encoded regulator. This scheme requires two further postulates: firstly, that tryptophan and $\mathrm{N}$ acetyltryptophan are readily interconverted only during conidiation; and secondly, that exogenous indoles induce their own breakdown and thereby lead to a shortage of 
AHT precursor in the conidiophores. G. Griffith (personal communication) has found evidence that exogenous indoles are degraded to anthranilic acid in conidiating cultures.

In scheme B, AHT is synthesized indirectly, via an unknown morphogenetic compound, and $b r l A$ acts independently of medA except in this step. This gives $N$ acetyltryptophan a unique role in conidiophore morphogenesis, and feedback controls of morphogen synthesis might explain the failure of exogenous indoles to enhance AHT synthesis.

At present both schemes depend on hypothetical features to explain morphology: morphogenetic loci in scheme $\mathrm{A}$ and a postulated morphogen in scheme B. In both schemes brlA plays a crucial regulatory role. It has been demonstrated that transcription of $i v o B$ is regulated, directly or indirectly, by brlA (Adams et al. 1988; Birse \& Clutterbuck, 1990 b). There is also good evidence that $b r l A$ encodes a DNA-binding protein; it has been sequenced and the inferred peptide contains a zincfinger DNA-binding motif (Boylan et al., 1987). Furthermore, mis-scheduled expression of $\mathrm{brlA}$ is sufficient to activate transcription of many genes characteristic of later conidiophore development, including ivoB (Adams et al., 1988). brlA and ivo $A$ or $-B$ mutants complement well in heterokaryons, implying that the $b r l A$ product is not confined to the nucleus which codes for it (cf. Cove, 1979; Scazzocchio et al., 1982).

The presence of AHTase in brlA14 and 19, which show no signs of $\mathrm{BRL}^{+}$morphology, implies that levels of $b r l A$ gene activity insufficient for morphogenesis can induce ivo $B$ at a low level. On the other hand, the fact that $\operatorname{brl} A$ mutants 14,19,10 (and 31, not shown here) possess AHTase but are unpigmented and have low AHT accumulation figures implies that the ivo $A$ gene (or possibly some other step in the AHT biosynthetic pathway) is inactive in these mutants. While there is a correlation between morphology and pigmentation, it is not complete: $b r l A 6$ is less developed but more pigmented than brlA10 (Clutterbuck, 1969). It therefore seems probable that if $b r l A$ acts as a transcriptional regulator, the BRLA proteins formed by various mutants have differing affinities for the promoters of $i v o A$ and $i v o B$, and for those of the genes governing morphogenesis. We are now cloning the ivo $A$ gene, and hope to be able to measure its transcription in $\mathrm{brlA}$ mutants and investigate the mechanism by which both ivo genes are induced.

I am grateful to Bill Timberlake, Charlie Birse, David Gems and Gareth Griffith for discussions on the material of this paper. Gareth has also confirmed some of the observations on the role of tryptophan as a source of AHT. I am also indebted, during the considerable time in which this study has been under way, for excellent assistance from June Baxendale, Eddie Campbell, Flora Roger and Moira Stark.

\section{References}

Adams, T. H., Boylan, M. T. \& Timberlake, W. E. (1988). brlA is necessary and sufficient to direct conidiophore development in Aspergillus nidulans. Cell 54, 353-362.

ANDERSON, S. O. (1985). Sclerotization and tanning of the cuticle. In Comprehensive Insect Physiology, Biochemistry and Pharmacology, vol. 3, pp. 59-74. Edited by G. A. Kerkut \& L. I. Gilbert. New York: Pergamon Press.

Bell, A. A. \& WheEler, M. H. (1986). Biosynthesis and function of fungal melanins. Annual Review of Phytopathology 24, 411-451.

Birse, C. E. \& ClutterbuCK, A. J. (1990a). N-Acetyl-6-hydroxytryptophan oxidase, a developmentally controlled phenol oxidase from Aspergillus nidulans. Journal of General Microbiology 136, 1725-1730.

Birse, C. E. \& ClutTERbUCK, A. J. (1990 b). Isolation and expression of the Aspergillus nidulans ivo $B$ gene coding for a developmental phenol oxidase. Gene (in the Press).

Boylan, M. T., Mirabito, P. M., Willett, C. E., Zimmerman, C. R. \& TIMBERLAKE, W. E. (1987). Isolation and physical characterization of three essential conidiation genes from Aspergillus nidulans. Molecular and Cellular Biology 7, 3113-3118.

Bull, A. T. \& CARTER, B. L. A. (1973). The isolation of tyrosinase from Aspergillus nidulans, its kinetic and molecular properties and some consideration of its activity in vivo. Journal of General Microbiology $75,61-73$.

ClutTERBUCK, A. J. (1969). A mutational analysis of conidial development in Aspergillus nidulans. Genetics 63, 317-327.

ClutTERBUCK, A. J. (1972). Absence of laccase from yellow-spored mutants of Aspergillus nidulans. Journal of General Microbiology 70 , 423-435.

Clutterbuck, A. J. (1974). Aspergillus nidulans. In Handbook of Genetics, vol. 1, pp. 447-510. Edited by R. C. King. New York: Plenum.

ClutterbuCK, A. J. (1977). The genetics of conidiation in Aspergillus nidulans. In The Physiology and Genetics of Aspergillus, pp. 305-317. Edited by J. E. Smith \& J. A. Pateman. London: Academic Press.

ClutTerbuck, A. J. (1978). Genetics of vegetative growth and asexual reproduction. In The Filamentous Fungi. III Developmental Biology, pp. 240-256. Edited by J. E. Smith \& D. R. Berry. London: Edward Arnold.

Clutterbuck, A. J. (1981). Map order of ygA and adD. Aspergillus News Letter 15, 21.

ClutterbuCK, A. J. (1986). Glasgow stock list of Aspergillus strains 1986. Fungal Genetics Newsletter 33, 59-69.

ClutTERBUCK, A. J. (1990a). Genetics of asexual development. In The Developmental Biology of Filamentous Ascomycetes. Edited by N. D. Read \& D. Moore. Cambridge: Cambridge University Press (in the Press).

Clutterbuck, A. J. (1990b). Aspergillus nidulans. In Genetic Maps. Locus Maps of Complex Genomes, 5th edn, pp. 3.97-3. 108. Edited by S. J. O'Brien. Cold Spring Harbor, NY: Cold Spring Harbor Laboratory.

Cove, D. J. (1979). Genetic studies of nitrate assimilation in Aspergillus nidulans. Biological Reviews 54, 291-327.

Hankinson, O. (1974). Mutants of the pentose phosphate pathway in Aspergillus nidulans. Journal of Bacteriology 117, 1121-1130.

HermanN, T. E., Kurtz, M. B. \& Champe, S. P. (1983). Laccase localized in hulle cells and cleistothecial primordia of Aspergillus nidulans. Journal of Bacteriology 154, 955-964.

JEPSON, J. B. (1960). Indoles and related compounds. In Chromatography and Electrophoretic Techniques, vol. I, pp. 183-211. Edited by I. Smith. London: Heineman.

JePson, J. B., Zaltzman, P. \& Udenfriend, S. (1962). Microsomal hydroxylation of tryptamine, indoleacetic acid and related compounds, to 6-hydroxy derivatives. Biochimica et Biophysica Acta 62, 91-102.

Johnstone, I. L., Hughes, S. G. \& Clutterbuck, A. J. (1985). Cloning an Aspergillus nidulans developmental gene by transformation. EMBO Journal 4, 1307-1311.

KUo, M.-J. \& ALEXANDER, M. (1967). Inhibition of the lysis of fungi by melanins. Journal of Bacteriology 94, 624-629. 
KURTZ, M. B. \& ChAMPE, S. P. (1981). Dominant spore color mutants of Aspergillus nidulans defective in germination and sexual development. Journal of Bacteriology 148, 629-638.

KURTZ, M. B. \& CHAMPE, S. P. (1982). Purification of conidial laccase of Aspergillus nidulans. Journal of Bacteriology 151, 1338-1345.

MALMSTRÖM, B. G. \& RYDÉN, L. (1968). The copper-containing oxidases. In Biological Oxidations, pp. 415-438. Edited by T. P. Singer. John Wiley: New York.

MARBACH, I. \& MAYER, A. M. (1978). Formation of catechol oxidase in seed coats of Pisum elatus during seed maturation. Journal of Experimental Botany 29, 69-75.

Martinelli, S. D. \& Bainbridge, B. W. (1974). Phenol oxidases of Aspergillus nidulans. Transactions of the British Mycological Society 63, 361-370

McCorkindale, N. J., Hayes, D., Johnston, G. A. \& Clutterbuck, A. J. (1983). $N$-Acetyl-6-hydroxytryptophan, a natural substrate of a monophenol oxidase from Aspergillus nidulans. Phytochemistry 22, 1026-1028.

PolacheCK, I. \& Rosenberger, R. F. (1977). Aspergillus nidulans mutant lacking $\alpha-(1,3)$-glucan, melanin and cleistothecia. Journal of Bacteriology 132, 650-656.

Pontecorvo, G., Roper, J. A., Hemmons, L. M., Macdonald, K. D. \& Bufton, A. W. J. (1953). The genetics of Aspergillus nidulans. Advances in Genetics 5, 141-238.

SCAZzocchio, C., SDRIN, N. \& ONG, G. (1982). Positive regulation in a eukaryote: a study of the $u a Y$ gene of Aspergillus nidulans. I. Characterization of alleles, dominance and complementation studies, and a fine structure map of the $u a Y-o x p A$ cluster. Genetics 100 . 185-208.

Wright, P. J. \& Pateman, J. A. (1970). Ultraviolet-light sensitive mutants of Aspergillus nidulans. Mutation Research 9, 579-587.

YAGER, L. N., KURTZ, M. B. \& ChAMPE, S. P. (1982). Temperatureshift analysis of conidial development in Aspergillus nidulans. Developmental Biology 93, 92-103.

Yelton, M. M., Hamer, J. E., De Souza, E. R., Mullaney, E. J. \& TIMBERLAKE, W. E. (1983). Developmental regulation of the Aspergillus nidulans trpC gene. Proceedings of the National Academy of Sciences of the United States of America 80, 7576-7580. 